SLAC-PUB-95-7029

October 1995

\section{Bunch Heating By Coherent Synchrotron Radiation}

\section{S.A. Heifets, M. Zolotorev}

Stanford Linear Accelerator Center Stanford University, Stanford, CA 94309

Acute interest in picosecond bunches revived intcrest in the efiects of coherent synchrotron radiation (CSR) on bunch parameters. As it is well known ${ }^{[1]}$, short bunches can produce the CSR provided the rms bunch length $\sigma$ is sufficiently small:

$$
\frac{\pi \sigma}{2 a} \sqrt{\frac{\rho}{a}}<1
$$

where $a$ is of the order of beam pipe radius, and $\rho$ is local bend radius. Under this condition, the CSR can be generated in the range of frequencies

$$
\frac{\pi}{a} \sqrt{\frac{\rho}{a}}<\frac{\omega}{c}<\frac{2}{\sigma}
$$

The power loss per particle in this range of frequencies is proportional to the number of particle per bunch $N_{b}$ and may be quite large.

Considering effect of the CSR on bunch parameters one need to distinguish a single pass situation (as in linac compressors) from the steady state situation in storage rings.

In the first case, the main effect is particle energy loss correlated with their position $z$ in the bunch. The energy loss per unit time per particle in the limit

\footnotetext{
* Work supported by Department of Energy contract DE-AC03-76SF00515

Presented at Micro Bunch Workshop on Production, Measurement and Applications of Short Bunches of Electrons and Positrons in Linacs and Storage Rings, Upton L.I., New York, September 28-30, 1995
}

$a \rightarrow \infty$ is given ${ }^{[2]}$ for a Gaussian bunch by the expression

$$
\frac{d E}{c d t}=-\Lambda F(z / \sigma), \quad \Lambda=\sqrt{\frac{\pi}{2}} \frac{N_{b} r_{0} m c^{2}}{3^{1 / 3}\left(\rho \sigma^{2}\right)^{2 / 3}},
$$

where factor $F=0$ in the tail of a bunch, $z=-\sigma$, reaches the maximum value $F \simeq 1$ at the center of a bunch, and $F \simeq-0.3$ for $z \simeq \sigma$, corresponding to energy gain of a particle in the head of a bunch. The parameter $\Lambda$ can be estimated as the energy loss due to coherent radiation of $N_{b}$ photons with the energy $h \omega$ of each photon, $\omega \simeq c / \sigma$. The radiation occurs on average $\alpha_{0} \gamma\left(\omega / \omega_{c}\right)^{1 / 3}$ times per turn, where $\alpha_{0}=1 / 137$, and $\omega_{c}=(3 / 2)(c / \rho) \gamma^{3}$.

The $z$-dependence in Eq. (3) can be obtained noticing that the coherent energy loss of a particle located at the distance $z$ from a center of a bunch $(z>0$ in the head of a bunch) is given by a spectral density of one-particle synchrotron radiation $P(\omega)$ :

$$
\frac{d E}{d t}=N_{b} \int d \omega P(\omega) \rho^{2}(\omega) e^{i \omega z / c}
$$

The integral here is taken over the range of frequencies defined by Eq. (2).

Comparing this equation with the definition of the longitudinal wake field we get the impedance of the coherent synchrotron radiation in terms of $P(\omega)$ :

$$
Z_{C S R}(\omega)=Z_{0} \frac{\pi \rho}{e^{2}} P(\omega), \quad Z_{0}=377 \mathrm{Ohm} .
$$

The power $P(\omega)$ at small frequencies $\omega<\ll \omega_{c}$ is

$$
P(\omega)=\frac{e^{2}}{\pi \rho} \Gamma(2 / 3) 3^{1 / 6} n^{1 / 3},
$$

where $n=\omega \rho / c$ is the harmonic number, $\Gamma(2 / 3) 3^{1 / 6}=1.62$. For these frequencies the impedace is

$$
\frac{Z_{C S R}(n)}{n}=1.62 \frac{Z_{0}}{n^{2 / 3}}
$$

with the maximum value $Z_{C S R}(n) / n=277(a / \rho) \mathrm{Ohm}$ at the lower end of the frequency range given by Eq. (2). Eq. (3) follows from Eq. (4) or Eq. (5). The effect 
Eq. (9) is the steady-state solution of Fokker-Plank equation

$$
\frac{\partial f}{\partial t}+\{H, F\}=\frac{\partial}{\partial \delta}\left[D \frac{\partial f}{\partial \delta}+\gamma_{d} \delta f\right]
$$

with the temperature $T=D / \gamma_{d}$ defined by the ratio of the diffusion coefficient $D$, caused usually by quantum fluctuations of synchrotron radiation, to the damping coefficient $\gamma_{d}$, defined by the dependence of the radiated power on particle energy. The diffusion coefficient is given by the rms fluctuations

$$
\frac{1}{\Delta t} \sum_{\Delta t}\left[<\left(\frac{h \omega}{E}\right)^{2}>-<\frac{h \omega}{E}>^{2}\right]
$$

and the damping coefficient is given by the average

$$
\frac{1}{\Delta t} \sum_{\Delta t}<\frac{h \omega}{E}>
$$

The averaging can be done by integration with the spectral density of photons expressed in terms of the total radiated power per particle.

The rms fluctuations in a system of $N$ particles are equivalent to $N$ independent particles. Therefore, the diffusion coefficient is not affected by the coherent synchrotron radiation.

The average in Eq. (13) can be split in two terms: one of them gives an average energy loss per unit time and is of no interest to us provided it is compensated by the RF voltage. The second term, obtained by expansion of Eq. (9) over $\delta$, defines $\gamma_{d}$. Considering now the CSR, we calculate the expression in Eq. (13) using the total radiated power per particle including CSR proportional to $N_{b}$. The resulting expression can be split now in three terms. The first term gives a constant, describing the total energy loss. The second term describes variation of the loss along the bunch proportional to $N_{b}$. Averaged over the revolution time, it corresponds to the CSR wake field. The third term, obtained by expansion in $\delta$, is proportional to the average $\langle\delta\rangle$ and defines damping of coherent oscillations rather than damping $\gamma_{d}$ of finite size $a$ can be taken into account limiting the range of integration in Eq. (4) according to $\mathrm{Eq}$. (2).

The formation length of the coherent photons is:

$$
\left(\frac{\rho}{\gamma}\right)\left(\frac{\omega_{c}}{\omega}\right)^{1 / 3}
$$

It is of the order of $\sqrt{a \rho}$ at low end of coherent frequencies $\omega=\pi / a \sqrt{\rho / a}$, and of the order of $\rho(\sigma / \rho)^{1 / 3}$ at the upper end $\omega / c=2 / \sigma$. The formation length is small in comparison with the circumference of the ring in both cases, i.e. the coherent radiation is a local process.

The situation in storage rings, where synchrotron motion shuffles particles in a bunch, is more complicated. The effect of CSR is described usually ${ }^{[1]}$ by the wake field $W_{C S R}(z)$. This wake modifies self-consistent potential $U(z)$ and, according to Haissinskii equation, changes one-particle distribution function $f$ in the steady state,

$$
f(z, \delta)=\frac{1}{Z} e^{-H / T}
$$

Here, the Hamiltonian $H(z, \delta)$,

$$
H=\frac{\delta^{2}}{2}+U(z)
$$

depends on the position of a particle $z$ within a bunch, $\delta=\Delta p / p$, and $T$ is temperature, $T=\langle H\rangle=\left\langle\delta^{2}>\right.$. If $W_{C S R}$ is a function only on $z$, then it modifies $U(z)$ changing the bunch length, but does not change the energy spread, which remains equal to the temperature $T^{+)}$.

This conclusion is based on two assumptions: first, that the CSR does not change temperature $T$, and, second, that Hamiltonian depends on $\delta$ quadratically as in Eq. (10). We discuss these assumptions in this paper.

+) This argument belongs to $K$. Oide 


\section{DISCLAIMER}

Portions of this document may be illegible in electronic image products. Images are produced from the best available original document. 
$(\Lambda / E)^{2}$ ct. The total diffusion coefficient is modified by the dynamic diffusion and, as a result, the temperature of a bunch increases provided $\xi>1$.

The parameter $\xi$ depends on the nonlinearity $\omega^{\prime}$. The nonlinearity of the RF field is very small, of the order of $\omega^{\prime} \simeq\left(\omega_{R F} \sigma / 2 c\right)^{2}$. The nonlinearity produced by the wake fields of the ring is much larger and may be of the order of one.

Take, for illustration, $\alpha=10^{-3}, \sigma=100 \mu, N_{b}=10^{10}, E=1 \mathrm{GeV}, \rho=10 \mathrm{~m}$, $l=\rho$, and assume $\omega^{\prime} \simeq 1$. Then $\xi \simeq 1$. The purpose of this example is to demonstrate that the problem deserves further (maybe, numerical) study.

The dynamic diffusion discussed above follows not from low repetition rate of kicks, as it usually happens in standard models of stochastic systems, but is the result of large kicks (the energy loss of the order of RF voltage) caused by CSR. Kicks, and the phase advance due to one-particle synchrotron motion, are large enough to generate random motion.

It is worth noting, that the dynamic diffusion may be considered for geometric wake fields as well. The parameter $\xi$ depends in this case on a geometric parameter (of the order of a beam pipe radius for the geometric wakes, and of the order of RF wave length for the RF voltage) instead of the bunch length $\sigma$. It is, therefore, much smaller than in the estimate above, $\xi<<1$, and the dynamic diffusion does not take place.

Let us turn now to the second assumption mentioned above, the structure of the Hamiltonian in the form of Eq. (6). The conclusion that CSR wake does not change the rms $\left\langle\delta^{2}>\right.$ was based on the Haissinskii equation Eq. (9). For arbitrary dependence of the Hamiltonian on momentum, solution of Eq. (9) does not have the form Eq. (10), and the conclusion of constant $<\delta^{2}>$ is, generally speaking, not valid anymore. This is the case, for example, for the lattices with small $\alpha$ where the nonlinear dispersion is essential. As a result, the Hamiltonian contains a cubic term in $\delta$. The solution of the Fokker-Plank equation can be obtained in this case changing variables $z, \delta$ to canonical angle-action variables $\phi, J$ such that the new Hamiltonian of one-particle motion. The averaging of the second term over a revolution period ignores the localized character of the CSR mentioned above. In general, localized kicks may lead to diffusion similar to diffusion in dynamic systems under external periodic perturbation. To demonstrate that we consider a ring where CSR takes place only locally, for example, in a special section with a large aperture and the length $l$. One-particle synchrotron motion is described by equations

$$
\frac{d x}{d s}=p ; \quad \frac{d p}{d s}=-\frac{\omega_{s}^{2}}{c^{2}} x+a x^{2}+b x^{3}+\lambda F(\sigma x) \delta_{T}(s)
$$

where $x=z / \sigma, p=-\alpha \delta / \sigma, s=c t, \omega_{s}$ is the zero current synchrotron frequency, $\delta_{L}(s)=\delta(s+L)$ is a periodic delta-function with period $L=2 \pi R . F(x)$ is introduced in Eq. (3), and

$$
\lambda=\frac{\alpha l}{\sigma E} \Lambda
$$

The coefficients $a$, and $b$ are given by non-linearity of the RF field

$$
a=\frac{\omega_{s}^{2} \cot \psi_{R F}}{2 c^{2}}\left(\frac{\omega_{R F}}{c}\right) \sigma, \quad b=\frac{\omega_{s}^{2}}{6 \omega_{R F}}\left(\frac{\omega_{R F} \sigma}{c}\right)^{3} \frac{1}{c \sigma} .
$$

Changing variables $x, p$ to action-angle variables $\phi, J$, we write the new Hamiltonian in the form $H(J, \phi)=H_{0}(J)-U(J, \phi) \delta_{T}(s)$. The one turn map $J, \phi \rightarrow \bar{J}, \bar{\phi}$ approximately is

$$
\bar{\phi}=\phi+2 \pi R \Omega(\bar{J})-U_{J}^{\prime}+o\left(U^{2}\right) ; \quad \bar{J}=J+U_{\phi}^{\prime}
$$

where $\Omega(J)=d H_{0} / d J$ and $U_{\phi}^{\prime}=d U / d \phi$. As usual, stability of the map is defined by the eigen-values of the infinitesimal matrix of the transform Eq. (17). The condition of stability is

$$
\left|2 \pi R \Omega^{\prime} U_{\phi, \phi}^{\prime \prime}\right|<4 .
$$

Here $\Omega^{\prime}=d^{2} H / d J^{2}$. An estimate shows that stability condition is defined by the parameter

$$
\xi=\omega^{\prime} \frac{\alpha N_{b} r_{0}}{\gamma \sigma}\left(\frac{\rho}{\sigma}\right)^{4 / 3}\left(\frac{l}{\rho}\right) .
$$

For large $\xi>1$ the motion is unstable and can be described as diffusion $<\delta^{2}>=$ 
$H(J)$ depends only on $J$. The Fokker-Plank equation takes the form ${ }^{[3]}$

$$
\frac{\partial f}{\partial t}+\{H, F\}=\gamma_{d} \frac{\partial f}{\partial J}\left[T D \frac{\partial f}{\partial J}+J f\right] .
$$

with the temperature $T$ given by usual quantum fluctuations of synchrotron radiation, and the diffusion coefficient is defined by averaging over $\phi$,

$$
D=<\left(\frac{\partial z}{\partial \phi}\right)^{2}>
$$

The steady-state solution depends only on $J$, all azimuthal harmonics of the distribution function vanish with time:

$$
f(J)=\frac{1}{Z} e^{-\frac{1}{T} \int^{J} \frac{J d J}{D(J)}}
$$

The distribution function now can not be factorized as in Eqs. (9) and (10). Hence, by changing the potential $U(z)$ we change the rms $\left\langle\delta^{2}\right\rangle$ as well.

The new bunch temperature $\hat{T}$ is defined by averaging $H(J)$ with the distribution function $f, \hat{T}=\langle H>$,

$$
\hat{T}=T \int d J f(J) \frac{\partial}{\partial J}\left[\frac{D(J) H(J)}{J}\right] .
$$

However, it can be shown that if the transform to the action-angle variables is done only in the first order of perturbation theory, the rms $\left\langle\delta^{2}\right\rangle$ remains unchanged. Hence, the effect of the nonlinear momentum compaction factor is quadratic in $\alpha_{2}$ and its effect on the energy spread is small.

\section{Conclusion}

We discuss here effects, which define the steady-state rms energy spread of a microbunch in a storage ring. It is implied that the longitudinal microwave instability is controlled by low $\alpha$ lattice. In this case the coherent synchrotron radiation, if exists, may be the main factor defining the bunch temperature. Another effect comes from the fact that a nonlinear momentum compaction of such lattices makes Haissinskii equation not applicable, and the coherent synchrotron radiation may effect not only bunch lengthening but the energy spread as well.

\section{Acknowledgment}

We are thankful to K. Oide for fruitful discussions.

\section{REFERENCES}

1. R.L.. Warnock and P. Morton, Particle Accelerators, 25, pp. 113-151, 1990; R. L. Warnock, SLAC-PUB-5523, 1991

2. J.B. Murphy, S. Krinsky, R.L. Gluckstern, Longitudinal Wakefield for Synchrotron Radiation, PAC 95; Y. Derbenev, J. Rossbach, E. Saldin, V. Shiltsev, Radiative Microbunch Tail-Head Interaction, Preprint TESLA-FFI 95-05, 1995

3. J. Schonfeld, Ann. Phys, V. 160, p. 149, 1985

\section{DISCLAIMER}

This report was prepared as an account of work sponsored by an agency of the United States Government. Neither the United States Government nor any agency thereof, nor any of their employees, makes any warranty, express or implied, or assumes any legal liability or responsibility for the accuracy, completeness, or usefulness of any information, apparatus, product, or process disclosed, or represents that its use would not infringe privately owned rights. Reference herein to any specific commercial product, process, or service by trade name, trademark, manufacturer, or otherwise does not necessarily constitute or imply its endorsement, recommendation, or favoring by the United States Government or any agency thereof. The views and opinions of authors expressed herein do not necessarily state or reflect those of the United States Government or any agency thereof. 